\title{
Linear sets and MRD-codes arising from a class of scattered linearized polynomials
}

\author{
Giovanni Longobardi $^{1}$ [D $\cdot$ Corrado Zanella $^{1}$
}

Received: 23 July 2020 / Accepted: 30 December 2020 / Published online: 21 January 2021

(C) The Author(s) 2021

\begin{abstract}
A class of scattered linearized polynomials covering infinitely many field extensions is exhibited. More precisely, the $q$-polynomial over $\mathbb{F}_{q^{6}}, q \equiv 1(\bmod 4)$ described in Bartoli et al. (ARS Math Contemp 19:125-145, 2020) and Zanella and Zullo (Discrete Math 343:111800, 2020) is generalized for any even $n \geq 6$ to an $\mathbb{F}_{q}$-linear automorphism $\psi(x)$ of $\mathbb{F}_{q^{n}}$ of order $n$. Such $\psi(x)$ and some functional powers of it are proved to be scattered. In particular, this provides new maximum scattered linear sets of the projective line $\operatorname{PG}\left(1, q^{n}\right)$ for $n=8,10$. The polynomials described in this paper lead to a new infinite family of MRD-codes in $\mathbb{F}_{q}^{n \times n}$ with minimum distance $n-1$ for any odd $q$ if $n \equiv 0(\bmod 4)$ and any $q \equiv 1(\bmod 4)$ if $n \equiv 2(\bmod 4)$.
\end{abstract}

Keywords Linearized polynomial $\cdot$ Linear set $\cdot$ Subgeometry $\cdot$ Finite field $\cdot$ Finite projective space $\cdot$ Rank metric code $\cdot$ MRD-code

\section{Introduction and preliminaries}

Let $\mathbb{F}_{q^{n}}$ be the Galois field of order $q^{n}, q$ a prime power. An $\mathbb{F}_{q}$-linearized polynomial, or $q$-polynomial, over $\mathbb{F}_{q^{n}}$ is a polynomial of the form

$$
f(x)=\sum_{i=0}^{k} c_{i} x^{q^{i}} \in \mathbb{F}_{q^{n}}[x], \quad k \in \mathbb{N} .
$$

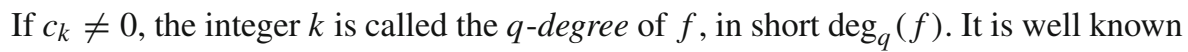
that any linearized polynomial defines an endomorphism of $\mathbb{F}_{q^{n}}$, when $\mathbb{F}_{q^{n}}$ is regarded

Giovanni Longobardi

giovanni.longobardi@unipd.it

Corrado Zanella

corrado.zanella@unipd.it

1 Dipartimento di Tecnica e Gestione dei Sistemi Industriali, Università degli Studi di Padova, Stradella S. Nicola, 3, 36100 Vicenza, VI, Italy 
as an $\mathbb{F}_{q}$-vector space and, vice versa, each element of $\operatorname{End}_{\mathbb{F}_{q}}\left(\mathbb{F}_{q^{n}}\right)$ can be represented as a unique linearized polynomial over $\mathbb{F}_{q^{n}}$ of $q$-degree less than $n$, see [17].

For a $q$-polynomial $f(x)=\sum_{i=0}^{n-1} c_{i} x^{q^{i}}$ over $\mathbb{F}_{q^{n}}$, let $D_{f}$ denote the associated Dickson matrix (or $q$-circulant matrix)

$$
D_{f}=\left(\begin{array}{cccc}
c_{0} & c_{1} & \ldots & c_{n-1} \\
c_{n-1}^{q} & c_{0}^{q} & \ldots & c_{n-2}^{q} \\
\vdots & \vdots & \vdots & \vdots \\
c_{1}^{q^{n-1}} & c_{2}^{q^{n-1}} & \ldots & c_{0}^{q^{n-1}}
\end{array}\right)
$$

The rank of the matrix $D_{f}$ is the rank of the $\mathbb{F}_{q}$-linear map $f(x)$, see [28].

Among the linearized polynomials over a finite field, a particular class has recently aroused interest for its connections with finite geometry and with coding theory: that of scattered polynomials.

More precisely, a scattered q-polynomial $f(x) \in \mathbb{F}_{q^{n}}[x]$ has the property that the polynomial $f(x)+m x$ has at most $q$ roots in $\mathbb{F}_{q^{n}}$ for all $m \in \mathbb{F}_{q^{n}}$, or equivalently, if for any $y, z \in \mathbb{F}_{q^{n}}^{*}$ the condition

$$
\frac{f(y)}{y}=\frac{f(z)}{z}
$$

implies that $y$ and $z$ are $\mathbb{F}_{q}$-linearly dependent. The condition for a $q$-polynomial to be scattered can be equivalently stated in terms of Dickson matrices [6,29]. Polynomials of this sort are linked to particular subsets of the finite projective line $\operatorname{PG}\left(1, q^{n}\right)$ called maximum scattered linear sets. Then consider the finite projective line $\Lambda=$ $\mathrm{PG}\left(\mathbb{F}_{q^{n}}^{2}, \mathbb{F}_{q^{n}}\right) \cong \mathrm{PG}\left(1, q^{n}\right)$. A set $L$ of points in $\Lambda$ is called $\mathbb{F}_{q}$-linear set (or just linear set) of rank $k$ if it consists of the points defined by the nonzero vectors of an $\mathbb{F}_{q}$-subspace $U$ of $\mathbb{F}_{q^{n}}^{2}$ of dimension $k$, i.e.

$$
L=L_{U}=\left\{\langle\mathbf{u}\rangle_{\mathbb{F}_{q^{n}}}: \mathbf{u} \in U \backslash\{\mathbf{0}\}\right\}
$$

Two linear sets $L_{U}$ and $L_{W}$ of $\mathrm{PG}\left(1, q^{n}\right)$ are said to be PГL-equivalent if there is an element $\varphi \in \operatorname{P\Gamma L}\left(2, q^{n}\right)$ such that $L_{U}^{\varphi}=L_{W}$. It is clear that if $U$ and $W$ are on the same $\Gamma \mathrm{L}\left(2, q^{n}\right)$-orbit, then $L_{U}$ and $L_{W}$ are P $\Gamma \mathrm{L}$-equivalent, but the converse statement is not true in general. For further details see $[7,11]$.

The set $L_{U}$ is called $\mathbb{F}_{q}$-linear set of $\mathcal{Z}(\Gamma L)$-class $r$ if $r$ is the greatest integer such that there exist $\mathbb{F}_{q}$-subspaces $U_{1}, U_{2}, \ldots, U_{r}$ of $\mathbb{F}_{q^{n}}^{2}$ such that $L_{U_{i}}=L_{U}$ for $i \in\{1,2, \ldots, r\}$ and $U_{i} \neq \lambda U_{j}$ for any $\lambda \in \mathbb{F}_{q^{n}}^{*}$ and distinct $i, j \in\{1,2, \ldots, r\}$. Furthermore, $L_{U}$ is of $\Gamma$ L-class $s$ if $s$ is the greatest integer such that there exist $\mathbb{F}_{q^{-}}$ subspaces $U_{1}, U_{2}, \ldots, U_{s}$ of $\mathbb{F}_{q^{n}}^{2}$ with $L_{U_{i}}=L_{U}$ for $i \in\{1,2, \ldots, s\}$, but $U_{i}$ and $U_{j}$ are not on the same $\Gamma \mathrm{L}\left(2, q^{n}\right)$-orbit for $i, j \in\{1,2, \ldots, s\}, i \neq j$. In particular, if $s=1$, then $L_{U}$ is called a simple $\mathbb{F}_{q}$-linear set.

The scattered $q$-polynomials arise from some $\mathbb{F}_{q}$-linear sets in $\operatorname{PG}\left(1, q^{n}\right)$. An $\mathbb{F}_{q^{-}}$ linear set of rank $k$ and size $\left(q^{k}-1\right) /(q-1)$ in $\mathrm{PG}\left(1, q^{n}\right)$ is called scattered. A scattered $\mathbb{F}_{q}$-linear set of rank $n$ is called maximum scattered linear set. As shown in 
[5], these are the linear sets of maximum size distinct from $\operatorname{PG}\left(1, q^{n}\right)$. If $L_{U}$ is an $\mathbb{F}_{q^{-}}$linear set of rank $n$ of $\mathrm{PG}\left(1, q^{n}\right)$, it can always be assumed (up to a projectivity) that $L_{U}$ does not contain the point $\langle(0,1)\rangle_{\mathbb{F}_{q^{n}}}$. Then, $U=U_{f}=\left\{(x, f(x)): x \in \mathbb{F}_{q^{n}}\right\}$, for some $q$-polynomial $f(x)$ over $\mathbb{F}_{q^{n}}$ and for the sake of simplicity, we will write $L_{f}$ instead of $L_{U_{f}}$ to denote the linear set defined by $U_{f}$. Clearly, $L_{f}$ is scattered if and only if $f(x)$ is a scattered $q$-polynomial. The first examples of scattered polynomials were found by Blokhuis and Lavrauw in [5] and by Lunardon and Polverino in [20] and then generalized by Sheekey in [26]. Apart from these, very few examples are known. They are defined for $n \leq 8$ and are summarized in Sect. 3. In view of the results in $[1,2]$, stating that the only families of scattered $q$-polynomials defined for infinitely many $n$ and satisfying certain additional assumptions are those of Blokhuis-Lavrauw and Lunardon-Polverino, it would seem that the scattered polynomials are quite rare.

As stated before, scattered polynomials attracted a lot of attention, especially because of their connection, established by Sheekey in [26, Section 5], with rank distance codes. These were introduced by Delsarte as $q$-analogs of the usual linear error- correcting codes endowed with Hamming distance, [13]. Recently, there has been a resurgence of interest in them because of their applications to random linear network coding and cryptography, see [14,27]. A rank distance code (or RD-code for short) $\mathcal{C}$ is a subset of the set of $m \times n$ matrices $\mathbb{F}_{q}^{m \times n}$ over $\mathbb{F}_{q}$, the finite field of $q$ elements with $q$ a prime power, endowed with the distance function

$$
d(A, B)=\operatorname{rk}(A-B)
$$

for any $A, B \in \mathbb{F}_{q}^{m \times n}$. The minimum distance of an $\mathrm{RD}$-code $\mathcal{C},|\mathcal{C}| \geq 2$, is defined as

$$
d(\mathcal{C})=\min _{\substack{M, N \in \mathcal{C} \\ M \neq N}} d(M, N)
$$

A rank distance code of $\mathbb{F}_{q}^{m \times n}$ with minimum distance $d$ has parameters $(m, n, q ; d)$. If $\mathcal{C}$ is an $\mathbb{F}_{q}$-linear subspace of $\mathbb{F}_{q}^{m \times n}$, then $\mathcal{C}$ is called $\mathbb{F}_{q}$-linear RD-code and its dimension $\operatorname{dim}_{\mathbb{F}_{q}} \mathcal{C}$ is defined to be the dimension of $\mathcal{C}$ as a subspace over $\mathbb{F}_{q}$.

The Singleton-like bound [13] for an $(m, n, q ; d) \operatorname{RD}$-code $\mathcal{C}$ is

$$
|\mathcal{C}| \leq q^{\max \{m, n\}(\min \{m, n\}-d+1)} .
$$

If the size of the code $\mathcal{C}$ meets this bound, then $\mathcal{C}$ is a called Maximum Rank Distance code, MRD-code for short. In this paper, only the case $m=n$ is considered; that is, only codes whose code words are square matrices are taken into account. Note that if $n=d$, then an MRD-code $\mathcal{C}$ consists of $q^{n}$ invertible endomorphisms of $\mathbb{F}_{q^{n}} ; \operatorname{such} \mathcal{C}$ is called spread set of $\operatorname{End}_{\mathbb{F}_{q}}\left(\mathbb{F}_{q^{n}}\right)$. In particular, if $\mathcal{C}$ is $\mathbb{F}_{q}$-linear, it is called a semifield spread set of $\operatorname{End}_{\mathbb{F}_{q}}\left(\mathbb{F}_{q^{n}}\right)$. These objects are related to semifields. For more details, see $[15,16]$.

The adjoint code of a rank code $\mathcal{C}$ is $\mathcal{C}^{\top}=\left\{C^{t}: C \in \mathcal{C}\right\}$, where $C^{t}$ denotes the transpose of the matrix $C$. Two $\mathbb{F}_{q}$-linear $\operatorname{codes} \mathcal{C}$ and $\mathcal{C}^{\prime}$ are called equivalent if there 
exist $P, Q \in \operatorname{GL}(n, q)$ and a field automorphism $\sigma$ of $\mathbb{F}_{q}$ such that

$$
\mathcal{C}^{\prime}=\left\{P C^{\sigma} Q: C \in \mathcal{C}\right\}
$$

Furthermore, $\mathcal{C}$ and $\mathcal{C}^{\prime}$ are weakly equivalent if $\mathcal{C}$ is equivalent to $\mathcal{C}^{\prime}$ or to $\left(\mathcal{C}^{\prime}\right)^{\top}$.

In general, it is difficult to decide whether two rank distance codes with the same parameters are equivalent or not. Useful tools to face this problem are the left and right idealizers, see [18,22]. More precisely, the left and right idealizers $L(\mathcal{C})$ and $R(\mathcal{C})$ of an $\mathrm{RD}$-code $\mathcal{C} \subseteq \mathbb{F}_{q}^{n \times n}$ are

$$
\begin{aligned}
& L(\mathcal{C})=\left\{X \in \mathbb{F}_{q}^{n \times n}: X C \in \mathcal{C} \text { for all } C \in \mathcal{C}\right\} \\
& R(\mathcal{C})=\left\{Y \in \mathbb{F}_{q}^{n \times n}: C Y \in \mathcal{C} \text { for all } C \in \mathcal{C}\right\}
\end{aligned}
$$

respectively.

In this article a class of scattered linearized polynomials over $\mathbb{F}_{q^{n}}$ will be introduced. Later, the connections to maximum scattered linear sets of the projective line and MRD-codes that arise from these polynomials will be investigated. More precisely, in Theorem 2.4, it will be proved that the polynomial

$$
2 \psi(x)=x^{q}+x^{q^{t-1}}-x^{q^{t+1}}+x^{q^{2 t-1}} \in \mathbb{F}_{q^{2 t}, \quad t \geq 3}
$$

is scattered for any odd $q$ if $t$ is even, and for $q \equiv 1(\bmod 4)$ if $t$ is odd. Some compositions of type $\psi \circ \psi \circ \cdots \circ \psi(x)$ are scattered as well. For $t=3, \psi(x)$ is up to equivalence the polynomial dealt with in $[4,30]$.

This paper is organized as follows. In Sect. 2, the polynomials of type (3) are investigated. In Sect. 3, it is shown that this family of scattered polynomials provides maximum scattered linear sets that are not of pseudoregulus type for any even $n \geq 6$. The related linear sets in $\mathrm{PG}\left(1, q^{n}\right)$ are proved to be PГL-equivalent to no previously known linear set for $n=8,10$.

In the last section, Sheekey's connection with the MRD-codes is described. In Theorem 5.4 it is proved that the class (3) of linearized polynomials gives rise to maximum subsets of square matrices of any even order $n \geq 6$ with minimum rank distance $d=n-1$. They are not equivalent to any previously known MRD-code.

\section{A class of scattered q-polynomials}

Throughout this paper, $q$ denotes a power of a prime $p \neq 2, t \geq 3, t \in \mathbb{N}$, and $n=2 t$. As usual, if $\ell$ divides $m$,

$$
\operatorname{Tr}_{q^{m} / q^{\ell}}(x)=x+x^{q^{\ell}}+x^{q^{2 \ell}}+\cdots+x^{q^{m-\ell}} \text { and } \quad \mathrm{N}_{q^{m} / q^{\ell}}(x)=x^{\frac{q^{m}-1}{q^{\ell}-1}}
$$


denote the trace and the norm of $x \in \mathbb{F}_{q^{m}}$ over $\mathbb{F}_{q^{\ell}}$. Consider the following $q$ polynomials in $\mathbb{F}_{q^{n}}[x]$ :

$$
\alpha_{n}(x)=\frac{\operatorname{Tr}_{q^{n} / q^{t}}(x)^{q^{t-1}}}{2} \text { and } \beta_{n}(x)=\frac{\left(x-x^{q^{t}}\right)^{q}}{2} .
$$

In the following, the index $n$ will usually be omitted: $\alpha(x)=\alpha_{n}(x), \beta_{n}(x)=\beta(x)$. Note that $\alpha(x)^{q^{t}}=\alpha(x), \beta(x)^{q^{t}}=-\beta(x)$ for any $x \in \mathbb{F}_{q^{n}}$.

Proposition 2.1 Let $\mathbb{F}_{q^{n}}$ be the finite field of order $q^{n}, n=2 t$, and consider

$$
W=\left\{x \in \mathbb{F}_{q^{n}}: x+x^{q^{t}}=0\right\} .
$$

Then,

(i) $\operatorname{ker} \alpha=\operatorname{im} \beta=W$;

(ii) $\operatorname{ker} \beta=\operatorname{im} \alpha=\mathbb{F}_{q^{t}}$;

(iii) the additive group of $\mathbb{F}_{q^{n}}$ is direct sum of $\mathbb{F}_{q^{t}}$ and $W$;

(iv) the product of two elements in $W$ is in $\mathbb{F}_{q^{t}}$;

(v) For any $k \in \mathbb{N}, q \equiv 1(\bmod 4)$, and $x \in \mathbb{F}_{q^{n}}, x^{q^{k}+1}=1$ implies $x \notin W$.

Proof Only the last statement is non-trivial. Let $\omega$ be a generator of the multiplicative group $\mathbb{F}_{q^{n}}^{*}$. Then,

$$
W=\left\{\omega^{(2 \ell+1)\left(q^{t}+1\right) / 2}: \ell=1,2, \ldots, q^{t}-1\right\} \cup\{0\} .
$$

In order to be a $\left(q^{k}+1\right)$-th root of the unity, the generic element of $W$ above must satisfy

$$
\frac{(2 \ell+1)\left(q^{t}+1\right)}{2}\left(q^{k}+1\right) \equiv 0 \quad\left(\bmod q^{2 t}-1\right),
$$

whence an integer $m$ exists satisfying

$$
2 m\left(q^{t}-1\right)=(2 \ell+1)\left(q^{k}+1\right) .
$$

Therefore, 4 must divide $q^{k}+1$. This implies that $q \equiv-1 \quad(\bmod 4)$, a contradiction.

Remark 2.2 If $t$ and $k$ are odd and $q \equiv 3(\bmod 4)$, then $\left(q^{t}-1\right) / 2$ is odd and $\left(q^{k}+1\right) / 4$ is an integer. This implies that (4) has a solution with $2 \ell+1=\left(q^{t}-1\right) / 2$ and $m=\left(q^{k}+1\right) / 4$. Therefore, an $x \in W$ exists such that $x^{q^{k}+1}=1$.

Now consider the $q$-polynomial

$$
\psi_{n}(x)=\alpha_{n}(x)+\beta_{n}(x) .
$$


The index $n$ will be often omitted in what follows.

For any positive integer,

$$
\psi^{(k)}(x)=\overbrace{\psi \circ \psi \circ \cdots \circ \psi}^{k}(x)
$$

will denote the $k$-fold composition of $\psi$ with itself. The polynomials $\alpha^{(k)}(x)$ and $\beta^{(k)}(x)$ are defined analogously. All $q$-polynomials will be considered here as maps; that is, they are reduced modulo $x^{q^{n}}-x$.

Proposition 2.3 The map $\psi(x)$ has order $n$.

Proof First, $\alpha^{(k)}(x)=\alpha(x)^{q^{(k-1)(t-1)}}$ and $\beta^{(k)}(x)=\beta(x)^{q^{k-1}}$ for any $k \in \mathbb{N}$, whence

$$
\alpha^{(n)}(x)=\operatorname{Tr}_{q^{n} / q^{t}}(x) / 2 \quad \text { and } \quad \beta^{(n)}(x)=\frac{x-x^{q^{t}}}{2}
$$

Note that for any $k \in \mathbb{N}$ and $x \in \mathbb{F}_{q^{n}}, \alpha^{(k)}(x) \in \mathbb{F}_{q^{t}}$ and $\beta^{(k)}(x) \in W$. Next, we prove by induction that for any $k \in \mathbb{N}$

$$
\psi^{(k)}(x)=\alpha^{(k)}(x)+\beta^{(k)}(x) .
$$

The induction base step $(k=1)$ is clear by the definition of $\psi(x)$. Now suppose that the property in (7) holds for $k-1$; then,

$$
\begin{aligned}
\psi^{(k)}(x)= & \psi\left(\psi^{(k-1)}(x)\right)=\psi\left(\alpha^{(k-1)}(x)\right)+\psi\left(\beta^{(k-1)}(x)\right) \\
& =\alpha\left(\alpha^{(k-1)}(x)\right)+\beta\left(\alpha^{(k-1)}(x)\right)+\alpha\left(\beta^{(k-1)}(x)\right)+\beta\left(\beta^{(k-1)}(x)\right) .
\end{aligned}
$$

By Proposition 2.1 (i) (ii), we get (7), that in view of (6) implies $\psi^{(n)}(x)=x$.

As a consequence of (7),

$$
\psi^{(k)}(x)=\frac{1}{2}\left(x^{q^{k}}+x^{q^{t-k}}-x^{q^{t+k}}+x^{q^{2 t-k}}\right)
$$

for any $0 \leq k \leq t$; (9) can be extended to any $k \in \mathbb{N}$ by considering modulo $2 t$ the exponents of $q$.

Theorem 2.4 Let $q$ be an odd prime power, $t \geq 3$, and

$$
\psi(x)=\frac{1}{2}\left(x^{q}+x^{q^{t-1}}-x^{q^{t+1}}+x^{q^{2 t-1}}\right) \in \mathbb{F}_{q^{2 t}}[x] .
$$

For $1 \leq k<2 t$, the $k$-fold composition $\psi^{(k)}(x)$ is scattered if and only if one of the following holds: $(i) t$ is even and $\operatorname{gcd}(k, t)=1$, or $(i i) t$ is odd, $\operatorname{gcd}(k, 2 t)=1$, and $q \equiv 1(\bmod 4)$ 
Proof The first part of the proof is devoted to prove that any of the conditions $(i)$ and (ii) implies that $\psi^{(k)}(x)$ is scattered. It is straightforward to see that the condition for $\psi^{(k)}(x)$ to be scattered can be rephrased in this way: if

$$
\psi^{(k)}(\rho x)=\rho \psi^{(k)}(x), \quad x, \rho \in \mathbb{F}_{q^{n}}, x \neq 0,
$$

then $\rho \in \mathbb{F}_{q}$. By Lemma 2.1 ( iii), for any $\rho \in \mathbb{F}_{q^{n}}$ there are precisely two elements $h=h_{\rho} \in \mathbb{F}_{q^{t}}$ and $r=r_{\rho} \in W$ such that $\rho=h+r$. Condition (10) is equivalent to

$$
\begin{aligned}
& \alpha^{(k)}\left((r+h)\left(x_{1}+x_{2}\right)\right)+\beta^{(k)}\left((r+h)\left(x_{1}+x_{2}\right)\right) \\
& =(r+h)\left(\alpha^{(k)}\left(x_{1}+x_{2}\right)+\beta^{(k)}\left(x_{1}+x_{2}\right)\right),
\end{aligned}
$$

with $x=x_{1}+x_{2}$, where $x_{1} \in \mathbb{F}_{q^{t}}$ and $x_{2}$ belongs to $W$.

By Proposition 2.1 ( $i$ ) (ii) (iv), the expression in (11) is reduced to

$$
\alpha^{(k)}\left(r x_{2}\right)+\alpha^{(k)}\left(h x_{1}\right)+\beta^{(k)}\left(r x_{1}\right)+\beta^{(k)}\left(h x_{2}\right)=(r+h)\left(\alpha^{(k)}\left(x_{1}\right)+\beta^{(k)}\left(x_{2}\right)\right) .
$$

Now, by expanding (12),

$$
\left(r x_{2}\right)^{q^{k(t-1)}}+\left(h x_{1}\right)^{q^{k(t-1)}}+\left(r x_{1}\right)^{q^{k}}+\left(h x_{2}\right)^{q^{k}}=(r+h)\left(x_{1}^{q^{k(t-1)}}+x_{2}^{q^{k}}\right) .
$$

Since $W$ and $\mathbb{F}_{q^{t}}$ meet in the trivial space, one obtains

$$
\left\{\begin{array}{l}
r x_{2}^{q^{k}}-r^{q^{k(t-1)}} x_{2}^{q^{k(t-1)}}=\left(h^{q^{k(t-1)}}-h\right) x_{1}^{q^{k(t-1)}} \\
r^{q^{k}} x_{1}^{q^{k}}-r x_{1}^{q^{k(t-1)}}=\left(h-h^{q^{k}}\right) x_{2}^{q^{k}}
\end{array}\right.
$$

Raising the first equation to its $q^{k}$-power,

$$
\left\{\begin{array}{l}
r^{q^{k}} x_{2}^{q^{2 k}}-r x_{2}=\left(h-h^{q^{k}}\right) x_{1} \\
r^{q^{k}} x_{1}^{q^{k}}-r x_{1}^{q^{k(t-1)}}=\left(h-h^{q^{k}}\right) x_{2}^{q^{k}}
\end{array}\right.
$$

This can be seen as a linear system in the unknowns $r$ and $r^{q^{k}}$. In the following, it will be assumed that $r \neq 0$, leading to a contradiction.

- Case 1. $x_{1}=0$ : the linear system in (14) is reduced to

$$
\left\{\begin{array}{l}
r^{q^{k}} x_{2}^{q^{2 k}}-r x_{2}=0 \\
\left(h-h^{q^{k}}\right) x_{2}^{q^{k}}=0
\end{array}\right.
$$


Since $x_{2} \neq 0$, the first equation in (15) gives $r^{q^{k}-1}=\left(x_{2}^{-1}\right)^{q^{2 k}-1}$. Then, there exists $\mu \in \mathbb{F}_{q^{k}}^{*} \cap \mathbb{F}_{q^{n}}=\mathbb{F}_{q}^{*}$ such that

$$
r=\mu\left(x_{2}^{-1}\right)^{q^{k}+1}
$$

The right-hand side of the last equation belongs to $\mathbb{F}_{q^{t}}^{*}$, that is a contradiction.

- Case 2. $x_{2}=0$ : as before, the linear system in (14) is reduced to

$$
\left\{\begin{array}{l}
\left(h-h^{q^{k}}\right) x_{1}=0 \\
r^{q^{k}} x_{1}^{q^{k}}-r x_{1}^{q^{k(t-1)}}=0
\end{array}\right.
$$

Since $x_{1} \neq 0$, by the second equation in (16), the existence of a $\lambda \in \mathbb{F}_{q}^{*}$ follows such that $r=\lambda\left(x_{1}^{q^{k}}\right)^{q^{k(t-3)}+q^{k(t-4)}+\ldots+q^{k}+1}$, implying $r \in \mathbb{F}_{q^{t}}^{*}$, a contradiction.

Note that (again under the assumption $r \neq 0$ ) $h \notin \mathbb{F}_{q}$, since for $h \in \mathbb{F}_{q}$ the same arguments as above lead to a contradiction.

- Case 3. $x_{1} \neq 0 \neq x_{2}$. The first goal is to prove that the determinant $D=$ $x_{1}^{q^{k}} x_{2}-x_{1}^{q^{k(t-1)}} x_{2}^{q^{2 k}}$ is not zero.

If $t$ is even, $D$ cannot be zero, otherwise the existence of a $\lambda \in \mathbb{F}_{q^{2}}$ would follow such that $x_{2}=\lambda\left(x_{1}^{-q^{k}}\right)^{q^{k(t-4)}+\ldots+q^{2 k}+1}$, implying $x_{2} \in \mathbb{F}_{q^{t}}$, a contradiction.

If $t$ is odd, $q \equiv 1(\bmod 4)$, and $D=0$, a necessary condition for (14) to have a solution is

$$
\operatorname{det}\left(\begin{array}{cc}
x_{2}^{q^{2 k}} & \left(h-h^{q^{k}}\right) x_{1} \\
x_{1}^{q^{k}} & \left(h-h^{q^{k}}\right) x_{2}^{q^{k}}
\end{array}\right)=0
$$

leading to $\left(x_{2}^{q^{k}} / x_{1}\right)^{q^{k}+1}=1$. Since $x_{2}^{q^{k}} / x_{1} \in W$, this contradicts Proposition $2.1(v)$.

Therefore, $D \neq 0$. Obtaining $r^{q^{k}}$ and $r$ from (14),

$$
\begin{gathered}
r^{q^{k}}=\left(h-h^{q^{k}}\right) \frac{x_{2}^{q^{k}+1}-x_{1}^{q^{k(t-1)}+1}}{x_{1}^{q^{k}} x_{2}-x_{1}^{q^{k(t-1)}} x_{2}^{q^{2 k}}} \text { and } \\
r=\left(h-h^{q^{k}}\right) \frac{x_{2}^{q^{2 k}+q^{k}}-x_{1}^{q^{k}+1}}{x_{1}^{q^{k}} x_{2}-x_{1}^{q^{k(t-1)}} x_{2}^{q^{2 k}}}
\end{gathered}
$$


Therefore,

$$
r^{q^{k}-1}=\frac{x_{2}^{q^{k}+1}-x_{1}^{q^{k(t-1)}+1}}{x_{2}^{q^{2 k}+q^{k}}-x_{1}^{q^{k}+1}}
$$

Note that $y=x_{2}^{q^{2 k}+q^{k}}-x_{1}^{q^{k}+1}$ belongs to $\mathbb{F}_{q^{t}}$, and $r^{q^{k}-1}=y^{q^{k(t-1)}-1}$. Then, there exists $\lambda \in \mathbb{F}_{q}$ such that $r=\lambda y^{q^{k(t-2)}+q^{k(t-3)}+\cdots+q^{k}+1}$, implying $r \in \mathbb{F}_{q^{t}}$, a contradiction again.

Hence, the condition $r \neq 0$ yields in all cases a contradiction. Taking into account (14), $h$ must belong to $\mathbb{F}_{q}$, implying $\rho \in \mathbb{F}_{q}$. This concludes the proof of the sufficiency.

If $d=\operatorname{gcd}(k, t) \neq 1$, then $\psi^{(k)}(x)$ is a $q^{d}$-polynomial; hence, it is not scattered as a $q$-polynomial. So, $\operatorname{gcd}(k, t)=1$ is a necessary condition.

Assume $k$ is even; then, it may be assumed that $t$ is odd. An $x \in \mathbb{F}_{q^{2}}^{*} \subseteq \mathbb{F}_{q^{n}}$ belongs to $W$ if and only if $x^{q-1}=-1$ which is an equation admitting $(q-1)$ solutions. Then fix $\mu \in W \cap \mathbb{F}_{q^{*}} \subseteq \mathbb{F}_{q^{n}}$ and $x_{2} \in W \backslash\{0\}$ arbitrarily. A solution of (14) is $x_{1}=h=0$, $r=\mu\left(x_{2}^{-1}\right)^{q^{k}+1}$, and this implies that $\psi^{(k)}(x)$ is not scattered.

For odd $t$ and $k$ and $q \equiv 3(\bmod 4)$, the $q$-polynomial $\psi^{(k)}(x)$ is not scattered. Indeed, taking $x_{1}=1, x_{2} \in W$ such that $x_{2}^{q^{k}+1}=1$, the equations in (14) are equivalent for any $h \in \mathbb{F}_{q^{t}}$. The images of the $\mathbb{F}_{q}$-linear maps $r \in W \mapsto r^{q^{k}} x_{2}^{q^{2 k}}-$ $r x_{2} \in \mathbb{F}_{q^{t}}$ and $h \in \mathbb{F}_{q^{t}} \mapsto\left(h-h^{q^{k}}\right) x_{1} \in \mathbb{F}_{q^{t}}$ both have $\mathbb{F}_{q}$-dimension at least $t-1$; this implies that their intersection is not trivial, and $r \in W, h \in \mathbb{F}_{q^{t}}$ exist such that $r \neq 0$ and (14) is satisfied.

Remark 2.5 Note that for $n=6,2 \psi^{(5)}(x)$ is the polynomial described in [30], that for $q \equiv 1(\bmod 4)$ and it is associated with the scattered $\mathbb{F}_{q}$-linear set $L_{h}^{5,6} \subseteq \operatorname{PG}\left(1, q^{6}\right)$, $h^{2}=-1$ that will be described in Sect. 3. Furthermore, $\psi^{(5)}(x)$ and $\psi(x)$ determine the same linear set, since $\psi^{(5)}(x)$ is the adjoint map of $\psi(x)$ with respect to the bilinear form $\operatorname{Tr}_{q^{6} / q}(x y)$ (cf. [3,7]).

\section{On the equivalence issue for linear sets}

By definition,

$$
L_{\psi_{n}^{(k)}}=\left\{\left\langle\left(x, \psi_{n}^{(k)}(x)\right)\right\rangle_{\mathbb{F}_{q^{n}}}: x \in \mathbb{F}_{q^{n}}^{*}\right\}
$$

denotes the maximum scattered $\mathbb{F}_{q}$-linear set of $\operatorname{PG}\left(1, q^{n}\right)$ associated with $\psi_{n}^{(k)}(x) \in$ $\mathbb{F}_{q^{n}}[x]$, provided that the assumptions of Theorem 2.4 are satisfied. The related $\mathbb{F}_{q^{-}}$ vector subspace of $\mathbb{F}_{q^{n}}^{2}$ is

$$
U_{\psi_{n}^{(k)}}=\left\{\left(x, \psi_{n}^{(k)}(x)\right): x \in \mathbb{F}_{q^{n}}\right\}
$$


Since the collineation $(a, b) \in \mathbb{F}_{q^{n}}^{2} \mapsto\left(a^{p}, b^{p}\right) \in \mathbb{F}_{q^{n}}^{2}$ stabilizes $U_{\psi_{n}^{(k)}}$, an $\mathbb{F}_{q}$-subspace of $\mathbb{F}_{q^{n}}^{2}$ is $\Gamma \mathrm{L}\left(2, q^{n}\right)$-equivalent to $U_{\psi_{n}}^{(k)}$ if and only if it is $\operatorname{GL}\left(2, q^{n}\right)$ equivalent to $U_{\psi_{n}^{(k)}}$.

Proposition 3.1 Let $t \geq 3$ and assume that $\psi_{n}^{(k)}(x)$ and $\psi_{n}^{(m)}(x)$ are scattered, $1 \leq$ $k, m<2 t=n$. Then, the $\mathbb{F}_{q^{-s u b s p a c e s}} U_{\psi_{n}^{(k)}}$ and $U_{\psi_{n}^{(m)}}$ are equivalent under the action of $\Gamma \mathrm{L}\left(2, q^{n}\right)$ if and only if $k=m$ or $k=n-m$.

\section{Proof Since}

$$
U_{\psi_{n}^{(k)}}=\left\{\left(\psi_{n}^{(n-k)}(x), x\right): x \in \mathbb{F}_{q^{n}}\right\}
$$

$U_{\psi_{n}^{(k)}}$ and $U_{\psi_{n}^{(n-k)}}$ are equivalent under the action of $\Gamma \mathrm{L}\left(2, q^{n}\right)$. This allows to prove the necessity of the condition only for $1 \leq k<m<t$.

Assume that $\left(\begin{array}{ll}a & b \\ c & d\end{array}\right)$ is an invertible matrix in $\mathbb{F}_{q^{n}}^{2 \times 2}$ such that for any $x \in \mathbb{F}_{q^{n}}$, a $z \in \mathbb{F}_{q^{n}}$ exists such that

$$
\left(\begin{array}{ll}
a & b \\
c & d
\end{array}\right)\left(\begin{array}{c}
x \\
\alpha^{(k)}(x)+\beta^{(k)}(x)
\end{array}\right)=\left(\begin{array}{c}
z \\
\alpha^{(m)}(z)+\beta^{(m)}(z)
\end{array}\right) .
$$

This implies

$$
\begin{aligned}
& c x+d \alpha^{(k)}(x)+d \beta^{(k)}(x) \\
& =\alpha^{(m)}\left(a x+b \alpha^{(k)}(x)+b \beta^{(k)}(x)\right)+\beta^{(m)}\left(a x+b \alpha^{(k)}(x)+b \beta^{(k)}(x)\right)
\end{aligned}
$$

for any $x \in \mathbb{F}_{q^{n}}$. Decompose any $x \in \mathbb{F}_{q^{n}}$ as a sum $x=x_{1}+x_{2}$ with $x_{1} \in \mathbb{F}_{q^{t}}$, $x_{2} \in W$. The above equation splits in

$$
\left\{\begin{array}{l}
c_{1} x_{1}+c_{2} x_{2}+d_{1} x_{1}^{q^{t-k}}+d_{2} x_{2}^{q^{k}}=\left(a_{1} x_{1}+a_{2} x_{2}+b_{1} x_{1}^{q^{t-k}}+b_{2} x_{2}^{q^{k}}\right)^{q^{t-m}} \\
c_{2} x_{1}+c_{1} x_{2}+d_{2} x_{1}^{q^{t-k}}+d_{1} x_{2}^{q^{k}}=\left(a_{2} x_{1}+a_{1} x_{2}+b_{2} x_{1}^{q^{t-k}}+b_{1} x_{2}^{q^{k}}\right)^{q^{m}}
\end{array}\right.
$$

Putting $x_{2}=0$ in (18), one obtains that for any $x_{1} \in \mathbb{F}_{q^{t}}$

$$
\left\{\begin{array}{l}
c_{1} x_{1}+d_{1} x_{1}^{q^{t-k}}-a_{1}^{q^{t-m}} x_{1}^{q^{t-m}}-b_{1}^{q^{t-m}} x_{1}^{q^{n-k-m}}=0 \\
c_{2} x_{1}+d_{2} x_{1}^{q^{t-k}}-a_{2}^{q^{m}} x_{1}^{q^{m}}-b_{2}^{q^{m}} x_{1}^{q^{t+m-k}}=0 .
\end{array}\right.
$$

Similarly,

$$
\left\{\begin{array}{l}
c_{2} x_{2}+d_{2} x_{2}^{q^{k}}-a_{2}^{q^{t-m}} x_{2}^{q^{t-m}}-b_{2}^{q^{t-m}} x_{2}^{q^{t+k-m}}=0 \\
c_{1} x_{2}+d_{1} x_{2}^{q^{k}}-a_{1}^{q^{m}} x_{2}^{q^{m}}-b_{1}^{q^{m}} x_{2}^{q^{k+m}}=0
\end{array}\right.
$$


must hold for any $x_{2} \in W$. After reducing modulo $x^{q^{t}}-x$ in (19) and modulo $x^{q^{t}}+x$ in (20), four polynomials are obtained all whose coefficients must be zero.

If $k+m \neq t$, the first identity in (19) implies $a_{1}=b_{1}=c_{1}=d_{1}=0$, and the first of (20) implies $a_{2}=b_{2}=c_{2}=d_{2}=0$, a contradiction.

Finally, assume $k+m=t$. The second of (19) gives $b_{2}=c_{2}=0$ and $d_{2}=a_{2}^{q^{m}}$; the first of (20) implies $d_{2}=a_{2}^{q^{t-m}}$. As a result, $a_{2}^{q^{2 m}-1}=-1 ; a_{2} \in \mathbb{F}_{q^{4 m}}^{*} \cap \mathbb{F}_{q^{2 t}}$. Since $m$ and $2 t$ are relatively prime, $a_{2} \in \mathbb{F}_{q^{4}} \backslash \mathbb{F}_{q^{2}}$, whence $t \equiv 2(\bmod 4)$. The first of (19) gives $a_{1}=d_{1}=0$ and $c_{1}=b_{1}^{q^{t-m}}$; the second of (20) implies $c_{1}=-b_{1}^{q^{m}}$. As a result, $b_{1}^{q^{2 m}-1}=-1$; so, $b_{1} \in \mathbb{F}_{q^{4}} \cap \mathbb{F}_{q^{t}}=\mathbb{F}_{q^{2}}$ and this is a contradiction.

A question that remains open is whether $L_{\psi_{n}^{(k)}}$ and $L_{\psi_{n}^{(m)}}$ are PГL-equivalent for $1 \leq k<m<t$.

In order to decide whether the linear sets $L_{\psi_{n}^{(k)}}$ are new, they will be compared to the known maximum scattered linear sets in $\mathrm{PG}\left(1, q^{n}\right)$. The known nonequivalent (under the action of $\Gamma \mathrm{L}\left(2, q^{n}\right)$ ) maximum scattered subspaces of $\mathbb{F}_{q^{n}}^{2}$, i.e. subspaces defining maximum scattered linear sets, are listed below.

1. $U_{s}^{1, n}=\left\{\left(x, x^{q^{s}}\right): x \in \mathbb{F}_{q^{n}}\right\}, 1 \leq s \leq n-1, \operatorname{gcd}(s, n)=1[5,12]$,

2. $U_{s, \delta}^{2, n}=\left\{\left(x, \delta x^{q^{s}}+x^{q^{n-s}}\right): x \in \mathbb{F}_{q^{n}}\right\}, n \geq 4, \mathrm{~N}_{q^{n} / q}(\delta) \notin\{0,1\}, \operatorname{gcd}(s, n)=1$ $[20,23,26]$,

3. $U_{s, \delta}^{3, n}=\left\{\left(x, \delta x^{q^{s}}+x^{q^{s+n / 2}}\right): x \in \mathbb{F}_{q^{n}}\right\}, n \in\{6,8\}, \operatorname{gcd}(s, n / 2)=$ $1, \mathrm{~N}_{q^{n} / q^{n / 2}}(\delta) \notin\{0,1\}$, for some $\delta$ and $q[9]$,

4. $U_{\delta}^{4,6}=\left\{\left(x, x^{q}+x^{q^{3}}+\delta x^{q^{5}}\right): x \in \mathbb{F}_{q^{6}}\right\}, q$ odd and $\delta^{2}+\delta=1$, see [10] for $q \equiv 0, \pm 1(\bmod 5)$, and [24] for the remaining congruences of $q$,

5. $U_{h}^{5,6}=\left\{\left(x, h^{q-1} x^{q}-h^{q^{2}-1} x^{q^{2}}+x^{q^{4}}+x^{q^{5}}\right): x \in \mathbb{F}_{q^{6}}, h \in \mathbb{F}_{q^{6}}, h^{q^{3}+1}=-1\right.$, $q$ odd $[4,30]$.

To make notation easier, $L_{s}^{i, n}, L_{s, \delta}^{i, n}, L_{\delta}^{4,6}$, and $L_{h}^{5,6}$ will denote the $\mathbb{F}_{q}$-linear sets defined by $U_{s}^{i, n}, U_{s, \delta}^{i, n}, U_{\delta}^{4,6}$, and $U_{h}^{5,6}$, respectively. Moreover, the sets $L_{s}^{1, n}$ and $L_{s, \delta}^{2, n}$ are called of pseudoregulus type and LP-type, respectively. As noted in the previous section, $L_{\psi_{6}}=L_{h}^{5,6}$ where $h^{2}=-1$ for $q \equiv 1 \quad(\bmod 4)$. In order to understand whether, under the assumptions of Theorem 2.4, the maximum scattered linear set $L_{\psi_{n}^{(k)}}$ is of pseudoregulus type or of LP type, some preliminary results have to be retraced.

First of all, Lunardon and Polverino in [21, Theorem 1 and 2], (see also [19]) showed that every linear set is projection of a canonical subgeometry, where a canonical subgeometry in $\mathrm{PG}\left(m-1, q^{n}\right)$ is a linear set $L$ of rank $m$ such that $\langle L\rangle=\mathrm{PG}(m-$ $\left.1, q^{n}\right)$. In particular, this result in the projective line case states that for each $\mathbb{F}_{q^{-}}$ linear set $L_{U}$ of the projective line $\Lambda=\operatorname{PG}\left(1, q^{n}\right)$ of rank $n$ there exists a canonical subgeometry $\Sigma=\operatorname{PG}(n-1, q)$ of $\Sigma^{*}=\operatorname{PG}\left(n-1, q^{n}\right)$, and an $(n-3)$-subspace $\Gamma$ of $\Sigma^{*}$ with $\Gamma$ disjoint from $\Sigma$ and $\Lambda$ such that

$$
L_{U}=\mathrm{p}_{\Gamma, \Lambda}(\Sigma)=\{\langle\Gamma, P\rangle \cap \Lambda: P \in \Sigma\}
$$


In [12], Csajbók and the second author gave a characterization of the linear sets of pseudoregulus type as a particular projection of a canonical subgeometry showing the following

Theorem 3.2 [12, Theorem 2.3] Let $\Sigma$ be a canonical subgeometry of $\mathrm{PG}\left(n-1, q^{n}\right)$, $q>2, n \geq 3$. Assume that $\Gamma$ and $\Lambda$ are an $(n-3)$-subspace and a line of $\mathrm{PG}\left(n-1, q^{n}\right)$, respectively, such that $\Gamma \cap \Sigma \neq \varnothing \neq \Gamma \cap \Lambda$. Then, the following assertions are equivalent:

(i) the set $\mathrm{p}_{\Gamma, \Lambda}(\Sigma)$ is a scattered $\mathbb{F}_{q}$-linear set of pseudoregulus type;

(ii) there exists a generator $\sigma$ of the subgroup $\mathrm{P} \Gamma \mathrm{L}\left(n, q^{n}\right)$ fixing $\Sigma$ pointwise and such that $\operatorname{dim}\left(\Gamma \cap \Gamma^{\sigma}\right)=n-4$;

(iii) there exist a point $P_{\Gamma}$ and a generator $\sigma$ of the subgroup of $\operatorname{P\Gamma L}\left(n, q^{n}\right)$ fixing $\Sigma$ pointwise, such that $\left\langle P_{\Gamma}, P_{\Gamma}^{\sigma}, \ldots, P_{\Gamma}^{\sigma^{n-1}}\right\rangle=\operatorname{PG}\left(n-1, q^{n}\right)$, and

$$
\Gamma=\left\langle P_{\Gamma}, P_{\Gamma}^{\sigma}, \ldots, P_{\Gamma}^{\sigma^{n-3}}\right\rangle
$$

Therefore, using the Theorem above, one obtains

Proposition 3.3 For any $n \geq 6$ and $k$ such that $\operatorname{gcd}(n, k)=1$, the linear set $L_{\psi_{n}}^{(k)}$ is not of pseudoregulus type.

Proof Since the linear set $L_{2 \psi_{n}^{(k)}}$ can be represented as the projection of the subgeometry $\Sigma$ whose points are of type $P_{u}=\left\langle\left(u, u^{q}, \ldots, u^{q^{n-1}}\right)\right\rangle_{\mathbb{F}_{q^{n}}}, u \neq 0$, from the vertex $\Gamma_{k}$ of equations $x_{0}=x_{k}+x_{t-k}-x_{t+k}+x_{n-k}=0$ onto the line $\ell$ of equations $x_{1}=x_{2}=\ldots=x_{n-k-1}=x_{n-k+1}=\ldots=x_{n-1}=0$. For, the hyperplane of $\mathrm{PG}\left(n-1, q^{n}\right)$ joining $\Gamma_{k}$ and $P_{u}$ has equations

$$
2 \psi_{n}^{(k)}(u) x_{0}-u\left(x_{k}+x_{t-k}-x_{t+k}+x_{n-k}\right)=0 .
$$

Such hyperplane meets $\ell$ in the point all whose coordinates are zero except $x_{0}=u$, $x_{n-k}=2 \psi_{n}^{(k)}(u)$. Let

$$
\sigma:\left\langle\left(x_{0}, x_{1}, \ldots, x_{n-1}\right)\right\rangle_{\mathbb{F}_{q^{n}}} \mapsto\left\langle\left(x_{n-1}^{q}, x_{0}^{q}, \ldots, x_{n-2}^{q}\right)\right\rangle_{\mathbb{F}_{q^{n}}},
$$

then $\sigma$ is a generator of the subgroup of $\operatorname{P\Gamma L}(n, q)$ fixing $\Sigma$ pointwise. Since the dimension of $\Gamma_{k} \cap \Gamma_{k}^{\sigma^{m}}$ is less than $n-4$ for any $m=1, \ldots, n-1, L_{\psi_{n}^{(k)}}$ is not of pseudoregulus type by Theorem 3.2.

In [30], the intersection number of an $(n-3)$-subspace $\Gamma$ of $\operatorname{PG}\left(n-1, q^{n}\right)$ with respect to a collineation $\sigma$ fixing pointwise a $q$-order subgeometry $\Sigma$ such that $\Sigma \cap \Gamma=$ $\emptyset$ has been defined as

$$
\operatorname{intn}_{\sigma}(\Gamma)=\min \left\{k \in \mathbb{N}: \operatorname{dim}\left(\Gamma \cap \Gamma^{\sigma} \cap \ldots \cap \Gamma^{\sigma^{k}}\right)>n-3-2 k\right\}
$$

By means of this notion and since the linear set $L_{s, \delta}^{2, n}$ has $\Gamma L$-class at most 2 for $n \in\{5,6,8\}$ (see [8] and [10]), Zullo and the second author gave a characterization in term of projection for linear sets of LP-type. More precisely, 
Theorem 3.4 [30, Theorem 3.5] Let L be a maximum scattered linear set in $\Lambda=$ $P G\left(1, q^{n}\right)$ with $n \leq 6$ or $n=8$. Then, $L$ is a linear set of LP-type if and only if for each $(n-3)$-subspace $\Gamma$ of $\mathrm{PG}\left(n-1, q^{n}\right)$ such that $L=\mathrm{p}_{\Gamma, \Lambda(\Sigma)}$, the following holds:

(i) there exists a generator $\sigma$ of the subgroup of $\mathrm{P} \Gamma \mathrm{L}\left(n, q^{n}\right)$ fixing $\Sigma$ pointwise, such that $\operatorname{int}_{\sigma}(\Gamma)=2$;

(ii) there exist a unique point $P$ and some point $Q$ of $\mathrm{PG}\left(n-1, q^{n}\right)$ such that

$$
\Gamma=\left\langle P, P^{\sigma}, \ldots, P^{\sigma^{n-4}}, Q\right\rangle
$$

and the line $\left\langle P^{\sigma^{n-1}}, P^{\sigma^{n-3}}\right\rangle$ meets $\Gamma$.

In [30, Section 5], exploiting the Theorem above, it has been shown that $L_{\psi_{6}}$ is not equivalent to a linear set of LP-type. The following proposition involves similar arguments. Clearly, by Proposition 3.1, hereafter one may suppose $k<t$.

Proposition 3.5 The linear set $L_{\psi_{8}^{(k)}}$ is not of LP-type for $k=1,3$.

Proof The result will be showed only for $k=1$. Similar considerations lead to the same result also for $k=3$. Then, as before, the linear set $L_{\psi_{8}^{(1)}}$ can be represented as the projection of the subgeometry $\Sigma$ whose points are of type $\left\langle\left(u, u^{q}, \ldots, u^{q^{7}}\right)\right\rangle_{\mathbb{F}_{q^{8}}}$, $u \neq 0$, from the vertex $\Gamma$ of equations $x_{0}=x_{1}+x_{3}-x_{5}+x_{7}=0$ onto the line $x_{1}=x_{2}=\ldots=x_{6}=0$. Let $\sigma \in \operatorname{P\Gamma L}\left(8, q^{8}\right)$ be defined as

$$
\left\langle\left(x_{0}, x_{1}, \ldots, x_{7}\right)\right\rangle_{\mathbb{F}_{q^{8}}}^{\sigma}=\left\langle\left(x_{1}^{q}, x_{2}^{q}, \ldots, x_{0}^{q}\right)\right\rangle_{\mathbb{F}_{q^{8}}} .
$$

The collineations $\sigma, \sigma^{3}, \sigma^{5}$ and $\sigma^{7}$ are the only generators of the subgroup of $\operatorname{P\Gamma L}\left(8, q^{8}\right)$ fixing pointwise the subgeometry $\Sigma$. Consider the subspaces

$$
\Gamma^{\sigma}:\left\{\begin{array}{l}
x_{1}=0 \\
x_{2}+x_{4}-x_{6}+x_{0}=0
\end{array} \text { and } \Gamma^{\sigma^{2}}:\left\{\begin{array}{l}
x_{2}=0 \\
x_{3}+x_{5}-x_{7}+x_{1}=0
\end{array}\right.\right.
$$

By direct computation, $\operatorname{dim}\left(\Gamma \cap \Gamma^{\sigma}\right)=3$ and, since $q$ is odd, $\operatorname{dim}\left(\Gamma \cap \Gamma^{\sigma} \cap \Gamma^{\sigma^{2}}\right)=1$.

Furthermore, since $\Gamma \cap \Gamma^{\sigma^{7}}=\left(\Gamma \cap \Gamma^{\sigma}\right)^{\sigma^{7}}$ and $\Gamma \cap \Gamma^{\sigma^{7}} \cap \Gamma^{\left(\sigma^{7}\right)^{2}}=\left(\Gamma \cap \Gamma^{\sigma} \cap \Gamma^{\sigma^{2}}\right)^{\sigma^{6}}$, we have $\operatorname{dim}\left(\Gamma \cap \Gamma^{\sigma^{7}}\right)=3$ and $\operatorname{dim}\left(\Gamma \cap \Gamma^{\sigma^{7}} \cap \Gamma^{\left(\sigma^{7}\right)^{2}}\right)=1$. Hence,

$$
\operatorname{intn}_{\sigma}(\Gamma)=\operatorname{intn}_{\sigma^{7}}(\Gamma) \geq 3
$$

A similar argument can be applied also for $\sigma^{3}$ and $\sigma^{5}$. As a consequence, the necessary condition stated in Theorem 3.4 for a linear set in $\mathrm{PG}\left(1, q^{8}\right)$ to be of LPtype is not satisfied by $L_{\psi_{8}^{(1)}}$.

In [9], the scattered subspace $U_{s, \delta}^{3, n}$ of $\mathbb{F}_{q^{n}}^{2}$ is exhibited for $n \in\{6,8\}, s$ coprime to $n$ and under some conditions on $\delta$ and $q$. 
Moreover, according to [9, Section 5], $U_{s, \delta}^{3, n}$ is $\operatorname{GL}\left(2, q^{n}\right)$-equivalent to $U_{n-s, \delta^{q^{n-s}}}^{3, n}$ and to $U_{s+n / 2, \delta^{-1}}^{3, n}$; thus, it is enough to take into account the linear sets $L_{s, \delta}^{3, n}$ with $s<n / 4, \operatorname{gcd}(s, n / 2)=1$ and hence only with $s=1$ for $n=6$, 8. Finally, the authors in [10, Proposition 4.1 and 4.2] showed that the $\mathcal{Z}(\Gamma L)$-class of $L_{1, \delta}^{3, n}$ is two and $L_{1, \delta}^{3, n}$ is a simple linear set.

Proposition 3.6 The linear set $L_{\psi_{8}^{(k)}}, k=1,3$, is not $\mathrm{P} \Gamma \mathrm{L}$-equivalent to $L_{s, \delta}^{3,8}$ for any $s$.

Proof By the results in $[9,10]$ quoted above, the linear set $L_{\psi_{8}^{(1)}}$ is PГL-equivalent to some $L_{s, \delta}^{3,8}$ if and only if

$$
U_{2 \psi}=\left\{\left(x, x^{q}+x^{q^{3}}-x^{q^{5}}+x^{q^{7}}\right): x \in \mathbb{F}_{q^{8}}\right\}
$$

is $\Gamma \mathrm{L}$-equivalent to $U_{1, \delta}^{3,8}$. Then suppose that there exist an invertible matrix $\left(\begin{array}{ll}a & b \\ c & d\end{array}\right)$ such that for each $x \in \mathbb{F}_{q^{8}}$, there exists $z \in \mathbb{F}_{q^{8}}$ satisfying

$$
\left(\begin{array}{ll}
a & b \\
c & d
\end{array}\right)\left(\begin{array}{c}
x \\
x^{q}+x^{q^{3}}-x^{q^{5}}+x^{q^{7}}
\end{array}\right)=\left(\begin{array}{c}
z \\
\delta z^{q}+z^{q^{5}}
\end{array}\right) .
$$

Equivalently, for each $x \in \mathbb{F}_{q^{8}}$,

$$
\begin{aligned}
c x & +d\left(x^{q}+x^{q^{3}}-x^{q^{5}}+x^{q^{7}}\right) \\
& =\delta\left[a^{q} x^{q}+b^{q}\left(x^{q^{2}}+x^{q^{4}}-x^{q^{6}}+x\right)\right]+\left[a^{q^{5}} x^{q^{5}}+b^{q^{5}}\left(x^{q^{6}}+x-x^{q^{2}}+x^{q^{4}}\right)\right] .
\end{aligned}
$$

This is a polynomial identity in $x$ that implies

$$
\left\{\begin{array}{l}
c=\delta b^{q}+b^{q^{5}} \\
d=\delta a^{q} \\
0=\delta b^{q}-b^{q^{5}} \\
d=0 \\
0=\delta b^{q}+b^{q^{5}} \\
d=-a^{q^{5}},
\end{array}\right.
$$

whence, since $\delta \neq 0, a=c=d=0$ : a contradiction. By applying the argument above for $U_{2 \psi_{8}^{(3)}}$, taking into account (9),

$$
\begin{aligned}
c x & +d\left(x^{q}+x^{q^{3}}+x^{q^{5}}-x^{q^{7}}\right) \\
& =\delta\left[a^{q} x^{q}+b^{q}\left(x^{q^{2}}+x^{q^{4}}+x^{q^{6}}-x\right)\right]+\left[a^{q^{5}} x^{q^{5}}+b^{q^{5}}\left(x^{q^{6}}+x+x^{q^{2}}-x^{q^{4}}\right)\right] .
\end{aligned}
$$


As before, this polynomial identity in $x$ implies

$$
\left\{\begin{array}{l}
c=-\delta b^{q}+b^{q^{5}} \\
d=\delta a^{q} \\
0=\delta b^{q}+b^{q^{5}} \\
d=0 \\
0=\delta b^{q}-b^{q^{5}} \\
d=a^{q^{5}}
\end{array}\right.
$$

whence, since $\delta \neq 0, a=c=d=0$, a contradiction again.

\section{$4 \mathcal{Z}(\Gamma \mathrm{L})$ - and $\Gamma L$-class of $L_{\psi_{n}^{(k)}}$ for some values of $n$ and $k$}

Now, similarly to what has been done in [7], the $\mathcal{Z}(\Gamma L)$-class and the $\Gamma$ L-class of the maximum scattered $\mathbb{F}_{q}$-linear set $L_{\psi_{n}^{(k)}}$ for small values of $n$ and $k$ will be determined. For the sake of completeness, the following preliminary results will be recalled.

Proposition 4.1 [10, Proposition 2.3] Let $f(x)$ and $g(x)$ be two q-polynomials over $\mathbb{F}_{q^{n}}$. Then, $L_{f} \subseteq L_{g}$ if and only if

$$
x^{q^{n}}-x \mid \operatorname{det} D_{F(Y)}(x) \in \mathbb{F}_{q^{n}}[x],
$$

where $F(Y)=f(x) Y-g(Y) x\left(c f\right.$. (1)). In particular, if $\operatorname{deg}\left(\operatorname{det} D_{F(Y)}(x)\right)<q^{n}$, then $L_{f} \subseteq L_{g}$ if and only if $\operatorname{det} D_{F(Y)}(x)$ is the zero polynomial.

Lemma 4.2 [7, Lemma 3.6] Let $f(x)=\sum_{i=0}^{n-1} a_{i} x^{q^{i}}$ and $g(x)=\sum_{i=0}^{n-1} b_{i} x^{q^{i}}$ be two q-polynomials over $\mathbb{F}_{q^{n}}$ such that $L_{f}=L_{g}$. Then,

$$
a_{0}=b_{0},
$$

for $k=1,2, \ldots, n-1$, it holds that

$$
a_{k} a_{n-k}^{q^{k}}=b_{k} b_{n-k}^{q^{k}}
$$

for $k=2,3, \ldots, n-1$, it holds that

$$
a_{1} a_{k-1}^{q} a_{n-k}^{q^{k}}+a_{k} a_{n-1}^{q} a_{n-k+1}^{q^{k}}=b_{1} b_{k-1}^{q} b_{n-k}^{q^{k}}+b_{k} b_{n-1}^{q} b_{n-k+1}^{q^{k}} .
$$

Therefore, the following results can be shown.

Proposition 4.3 Let $q \equiv 1(\bmod 4)$. The $\mathcal{Z}(\Gamma \mathrm{L})$-class of $L_{\psi_{6}}$ is two. Moreover, $L_{\psi_{6}}$ is a simple linear set. 
Proof Since $\psi_{6}(x)$ and $\psi_{6}^{(5)}(x)$ define the same linear set, we know that $L_{2 \psi_{6}}=L_{2 \psi_{6}^{(5)}}$.

Suppose $L_{\varphi}=L_{2 \psi_{6}}$ for some $\varphi(x)=\sum_{i=0}^{5} a_{i} x^{q^{i}} \in \mathbb{F}_{q^{6}}[x]$. We show that there exists $\lambda \in \mathbb{F}_{q^{6}}^{*}$ such that either $\lambda U_{\varphi}=U_{2 \psi_{6}}$ or $\lambda U_{\varphi}=U_{2 \psi_{6}^{(5)}}$.

By (24), (25) and (26) in Lemma 4.2, one obtains that

$$
a_{0}=a_{3}=0, \quad a_{1} a_{5}^{q}=1 \text { and } a_{2} a_{4}^{q^{2}}=-1 .
$$

By Proposition 4.1, the Dickson matrix associated with the $q$-polynomial

$$
F(Y)=\varphi(x) Y-2 \psi_{6}(Y) x
$$

has determinant $D_{F(Y)}(x)$ equal to zero for each $x \in \mathbb{F}_{q^{6}}$, where, by (27),

$$
\varphi(x)=a_{1} x^{q}+a_{2} x^{q^{3}}-a_{2}^{-q^{4}} x^{q^{4}}+a_{1}^{-q^{5}} x^{q^{5}} .
$$

Direct computation shows that

$$
D_{F(Y)}(x)=\frac{1}{\mathrm{~N}_{q^{6} / q}\left(a_{1} a_{2}\right)} Q_{a_{1}, a_{2}}(x),
$$

where $Q_{a_{1}, a_{2}}(x)$ is a polynomial in $\mathbb{F}_{q^{6}}[x]$ whose coefficients are polynomials in $a_{1}$ and $a_{2}$.

By a straightforward estimate, we note that the $\operatorname{deg}\left(Q_{a_{1}, a_{2}}(x)\right)$ is at most $4 q^{5}+$ $2 q^{4}$. Since $q \geq 5, \operatorname{deg}\left(Q_{a_{1}, a_{2}}(x)\right)$ is less than $q^{6}$. Therefore, $Q_{a_{1}, a_{2}}(x)$ is the null polynomial. Consider the coefficient

$$
a_{1}^{1+q+q^{2}} a_{2}^{1+q+2 q^{2}+q^{4}}\left(a_{1}^{q^{3}+q^{4}}-a_{2}^{q^{3}}\right)\left(a_{1}^{q^{3}+q^{4}}+a_{2}^{q^{3}}\right)
$$

of the term $x^{q^{3}+2 q^{4}+3 q^{5}}$ of $Q_{a_{1}, a_{2}}(x)$, it is zero if and only if either $a_{2}=a_{1}^{q+1}$ or $a_{2}=-a_{1}^{q+1}$. In both cases, since up to the sign the coefficient of the term $x^{q+q^{2}+q^{4}+3 q^{5}}$ is $a_{1}^{1+q+q^{2}+2 q^{3}+2 q^{4}}\left(\mathrm{~N}_{q^{6} / q}\left(a_{1}\right)-1\right)^{2}$ and it vanishes, we get $\mathrm{N}_{q^{6} / q}\left(a_{1}\right)=1$. Therefore, putting $a_{1}=\lambda^{q-1}$, we obtain $\lambda U_{\varphi}=U_{2 \psi_{6}}$ if $a_{2}=a_{1}^{q+1}$ and $\lambda U_{\varphi}=U_{2 \psi_{6}^{(5)}}$ if $a_{2}=-a_{1}^{q+1}$. Hence, the $\mathcal{Z}(\Gamma \mathrm{L})$-class of $L_{\psi_{6}}$ is two and, by Proposition 3.1 , it is simple.

Proposition 4.4 The $\mathcal{Z}(\Gamma \mathrm{L})$-class of $L_{\psi_{8}^{(k)}}, k=1,3$, is two. Moreover, $L_{\psi_{8}^{(k)}}$ is a simple linear set.

Proof First, we prove the statement for $k=1$. Since $\psi_{8}(x)$ and $\psi_{8}^{(7)}(x)$ define the same linear set, we know $L_{2 \psi_{8}}=L_{2 \psi_{8}^{(7)}}$. Suppose $L_{\varphi}=L_{2 \psi_{8}(x)}$ for some $\varphi(x)=$ $\sum_{i=0}^{7} a_{i} x^{q^{i}} \in \mathbb{F}_{q^{8}}[x]$. We show that there exists $\lambda \in \mathbb{F}_{q^{8}}^{*}$ such that either $\lambda U_{\varphi}=U_{2 \psi_{8}}$ or $\lambda U_{\varphi}=U_{2 \psi_{8}^{(7)}}$. 
By (24), (25) and (26) in Lemma 4.2, one obtains that

$$
a_{0}=a_{2}=a_{4}=a_{6}=0, \quad a_{1} a_{7}^{q}=1 \quad \text { and } \quad a_{3} a_{5}^{q^{3}}=-1
$$

By Proposition 4.1, the Dickson matrix associated with the $q$-polynomial

$$
F(Y)=\varphi(x) Y-2 \psi_{8}(Y) x
$$

has zero determinant $D_{F(Y)}(x)$ for each $x \in \mathbb{F}_{q^{8}}$, where, by (30),

$$
\varphi(x)=a_{1} x^{q}+a_{3} x^{q^{3}}-a_{3}^{-q^{5}} x^{q^{5}}+a_{1}^{-q^{7}} x^{q^{7}} .
$$

Direct computation shows that

$$
D_{F(Y)}(x)=\frac{1}{\mathrm{~N}_{q^{8} / q}\left(a_{1} a_{3}\right)} Q_{a_{1}, a_{3}}(x),
$$

where $Q_{a_{1}, a_{3}}(x)$ is a polynomial in $\mathbb{F}_{q^{8}}[x]$ whose coefficients are polynomials in $a_{1}$ and $a_{3}$. By a straightforward estimate, we note that the $\operatorname{deg}\left(Q_{a_{1}, a_{3}}(x)\right)$ is at most $4\left(q^{6}+q^{7}\right)$.

- Case $1, q \geq 5$. In this case, $4\left(q^{6}+q^{7}\right)$ is less than $q^{8}$. Therefore, $D_{F(Y)}(x)$ is the null polynomial. Consider then the coefficient $x^{q^{4}+q^{5}+3 q^{6}+3 q^{7}}$ of $Q_{a_{1}, a_{2}}(x)$, it is zero if and only if either $a_{3}=a_{1}^{q^{2}+q+1}$ or $a_{3}=-a_{1}^{q^{2}+q+1}$.

In both cases, then, since up to the sign the coefficient

$$
a_{1}^{2+q^{3}+2 q^{4}+2 q^{5}+3 q^{6}+4 q^{7}}\left(\mathrm{~N}_{q^{8} / q}\left(a_{1}\right)-1\right)^{2}
$$

of term $x^{3+3 q+q^{2}+q^{5}}$ is zero, $\mathrm{N}_{q^{8} / q}\left(a_{1}\right)=1$ follows.

Therefore, putting $a_{1}=\lambda^{q-1}$, we obtain $\lambda U_{\varphi}=U_{2 \psi_{8}}$ if $a_{3}=a_{1}^{q^{2}+q+1}$ and $\lambda U_{\varphi}=U_{2 \psi_{8}^{(7)}}$ if $a_{3}=-a_{1}^{q^{2}+q+1}$.

- Case $q=3$. Reducing the polynomial $Q_{a_{1}, a_{3}}(x)$ in (29) modulo ( $\left.x^{q^{8}}-x\right)$, then one gets that the coefficient of $x^{48}$ is $a_{1}^{5480} a_{3}^{4248}-a_{1}^{5454} a_{3}^{4250}$. Then, either $a_{3}=$ $a_{1}^{q^{2}+q+1}$ or $a_{3}=-a_{1}^{q^{2}+q+1}$. In both cases, since $Q_{a_{1}, a_{2}}(x)\left(\bmod x^{q^{8}}-x\right)$ has to be the null polynomial, up to sign the coefficient of term $x^{2439}$ is $a^{2860}\left(\mathrm{~N}_{q^{8}}\left(a_{1}\right)-1\right)^{2}$, whence $\mathrm{N}_{q^{8} / q}\left(a_{1}\right)=1$. Therefore, putting $a_{1}=\lambda^{q-1}$, we obtain $\lambda U_{\varphi}=U_{2 \psi_{8}}$ if $a_{3}=a_{1}^{q^{2}+q+1}$ and $\lambda U_{\varphi}=U_{2 \psi_{8}^{(7)}}$ if $a_{3}=-a_{1}^{q^{2}+q+1}$.

The computations for $k=3$ are similar and we omit to report them. Hence, the $\mathcal{Z}(\Gamma \mathrm{L})$-class of $L_{\psi_{8}^{(k)}}$ is two for $k=1,3$ and, by Proposition 3.1, such linear set is simple.

Corollary 4.5 The linear sets $L_{\psi_{8}}$ and $L_{\psi_{8}^{(3)}}$ are not PГL-equivalent. 
Proposition 4.6 Let $q \equiv 1(\bmod 4)$. The $\mathcal{Z}(\Gamma \mathrm{L})$-class of $L_{\psi_{10}}$ is two. Moreover, $L_{\psi_{10}}$ is a simple linear set.

Proof Like in the previous propositions, (24), (25) and (26) in 4.2 imply that if $L_{\varphi}=$ $L_{2 \psi_{10}(x)}$ for some $\varphi(x)=\sum_{i=0}^{9} a_{i} x^{q^{i}} \in \mathbb{F}_{q^{10}}[x]$, then

$$
a_{0}=a_{2}=a_{3}=a_{5}=a_{7}=a_{8}=0,
$$

and $a_{9}=a_{1}^{-q^{9}}, a_{6}=-a_{4}^{-q^{6}}$. The determinant $D_{F(Y)}(x)$ of the Dickson matrix associated with $\varphi(x) Y-\left(Y^{q}+Y^{q^{4}}-Y^{q^{6}}+Y^{q^{9}}\right) x$ has degree at most $4 q^{9}+4 q^{8}+2 q^{7}<$ $q^{10}$, so it vanishes. The coefficient of $x^{3+3 q+2 q^{2}+q^{3}+q^{4}}$ in $D_{F(Y)}(x)$ is

$$
a_{1}^{w_{1}} a_{4}^{w_{2}}\left(a_{1}^{2\left(1+q+q^{2}+q^{3}\right)}-a_{4}^{2}\right)
$$

for some $w_{1}, w_{2} \in \mathbb{Z}$, implying $a_{4}= \pm a_{1}^{1+q+q^{2}+q^{3}}$. In both cases, by substituting such expressions of $a_{4}$ in $D_{F(Y)}(x)$, the coefficient of $x q^{3}+3 q^{7}+3 q^{8}+3 q^{9}$ is

$$
\pm a_{1}^{w}\left(1-\mathrm{N}_{q^{10} / q}\left(a_{1}\right)\right)^{2}
$$

for some $w \in \mathbb{Z}$, whence $\mathrm{N}_{q^{10} / q}\left(a_{1}\right)=1$. The proof can now be completed as in Propositions 4.3 and 4.4.

Corollary 4.7 The linear set $L_{\psi_{10}}$ is a new maximum scattered $\mathbb{F}_{q}$-linear set in $\operatorname{PG}\left(1, q^{10}\right)(q \equiv 1(\bmod 4))$.

Proof Since $L_{\psi_{10}}$ is a simple linear set, it is enough to check that $U_{\psi_{10}}$ does not belong to the $\Gamma$ L-orbit of some $U_{s, \delta}^{2,10}$. This will be proved in Proposition 5.3 in a more general result.

Remark 4.8 For $n=10$ and $k=3$, the equations in Lemma 4.2 do not imply that six coefficients of $\varphi(x)$ are equal to zero, like in (33). This adds complexity to the computations.

It is not known to the authors of this paper whether $L_{\psi_{n}^{(k)}}$ is a new linear set for $n>10$ and $\operatorname{gcd}(n, k)=1$ or $n=10$ and $k=3$. Indeed, it would be necessary to show that $L_{\psi_{n}^{(k)}}$ is not a linear set of LP-type. Furthermore, the techniques used so far do not seem to be within easy reach when solving the issue of $\mathcal{Z}(\Gamma \mathrm{L})$ - and $\Gamma \mathrm{L}$-class of $L_{\psi_{n}^{(k)}}$ for $n>10$ and $\operatorname{gcd}(n, k)=1$ or $n=10$ and $k=3$.

The following result describes the intersection of $L_{\psi_{n}^{(k)}}$ with a special Baer subline.

Proposition 4.9 Assume that $\psi_{n}^{(k)}$ is a scattered q-polynomial, $1 \leq k<t$. Let $\Sigma \cong$ $\mathrm{PG}\left(1, q^{t}\right)$ be the subline of $\mathrm{PG}\left(1, q^{n}\right)$ consisting of the points represented by nonzero pairs in $\mathbb{F}_{q^{t}}^{2}$. Then, $\Sigma \cap L_{\psi_{n}^{(k)}}$ is partitioned into two $\mathbb{F}_{q}$-linear sets of pseudoregulus type of $\mathrm{PG}\left(1, q^{t}\right)$. 
Proof The points of an $\mathbb{F}_{q}$-linear set of pseudoregulus type contained in $L_{\psi_{n}^{(k)}}$ are of type $\left\langle\left(h, h^{q^{t-k}}\right)\right\rangle_{\mathbb{F}_{q^{n}}}$ for $h \in \mathbb{F}_{q^{t}}^{*}$. Let $\xi \in \mathbb{F}_{q^{t}}$ be such that $\mathrm{N}_{q^{t} / q}(\xi)=-1$. The map $\langle(a, b)\rangle_{\mathbb{F}_{q^{n}}} \mapsto\langle(a, \xi b)\rangle_{\mathbb{F}_{q^{n}}}$ induces a projectivity of $\Sigma$ mapping any of the $\left(q^{t}-\right.$ $1) /(q-1)$ points of type $\left\langle\left(r, r^{q^{k}}\right)\right\rangle_{\mathbb{F}_{q^{n}}}, r \in W \backslash\{0\}$ in a point having nonhomogeneous coordinate, say $\eta_{r}$, satisfying $\mathrm{N}_{q^{t} / q}\left(\eta_{r}\right)=1$. Therefore, $M=\left\{\left\langle\left(r, r^{q^{k}}\right)\right\rangle_{\mathbb{F}_{q^{n}}}: r \in\right.$ $W \backslash\{0\}\}$ is a further linear set of pseudoregulus type contained in $\Sigma$.

Next, let $P=\langle(u, v)\rangle_{\mathbb{F}_{q^{n}}}$ be a point in $\Sigma$, with $u, v \in \mathbb{F}_{q^{t}}$. Then, $P$ belongs to $L_{\psi_{n}^{(k)}}$ if and only if there exists $\lambda, x \in \mathbb{F}_{q^{n}}^{*}$ such that $x=\lambda u$ and $\psi^{(k)}(x)=\lambda v$. This is equivalent to

$$
\frac{\psi^{(k)}(x)}{x}=\frac{v}{u},
$$

whence $\psi^{(k)}(x) / x \in \mathbb{F}_{q^{t}}$. Equivalently,

$$
\left(\frac{\psi^{(k)}(x)}{x}\right)^{q^{t}}=\frac{\psi^{(k)}(x)}{x}
$$

that can be reformulated in

$$
\alpha^{(k)}(x) \beta(x)^{q^{2 t-1}}=\beta^{(k)}(x) \alpha(x)^{q} .
$$

Clearly, the equation is satisfied by all $x$ either in $\mathbb{F}_{q^{t}}$ or in $W$. Now suppose that $x \in \mathbb{F}_{q^{n}} \backslash\left(\mathbb{F}_{q^{t}} \cup W\right)$. Then, there exist $x_{1} \in \mathbb{F}_{q^{t}}$ and $x_{2} \in W$, both nonzero, such that $x=x_{1}+x_{2}$. Next, (34) implies

$$
\alpha^{(k)}\left(x_{1}\right) \beta\left(x_{2}\right)^{q^{2 t-1}}=\beta^{(k)}\left(x_{2}\right) \alpha\left(x_{1}\right)^{q} .
$$

This is equivalent to $x_{2}^{q^{k}-1}=x_{1}^{q^{k(t-1)}-1}$; therefore, there exists $\mu \in \mathbb{F}_{q}$ such that $x_{2}=\mu x_{1}^{q^{k(t-2)}+\ldots+1}$, a contradiction.

\section{New MRD-codes}

As recalled before, in [26, Section 5] Sheekey explicated a link between maximum scattered $\mathbb{F}_{q}$-linear sets of $\mathrm{PG}\left(1, q^{n}\right)$ and $\mathbb{F}_{q}$-linear MRD-codes with minimum distance $d=n-1$. We briefly describe such relationship. After fixing an $\mathbb{F}_{q}$-basis for $\mathbb{F}_{q^{n}}$, we can define an isomorphism between the $\operatorname{rings} \operatorname{End}_{\mathbb{F}_{q}}\left(\mathbb{F}_{q^{n}}\right)$ and $\mathbb{F}_{q}{ }^{n \times n}$ and then any RD-code can be seen as a subset of linearized polynomials over $\mathbb{F}_{q^{n}}$. Next,

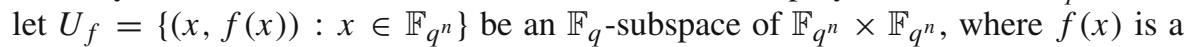
$q$-polynomial over $\mathbb{F}_{q^{n}}$. The set

$$
\mathcal{C}_{f}=\left\{a f(x)+b x: a, b \in \mathbb{F}_{q^{n}}\right\}=\langle x, f(x)\rangle_{q^{n}}
$$


corresponds to a subset of square matrices of order $n$ over $\mathbb{F}_{q}$ and hence to a rank distance code. In particular, the following result holds:

Theorem 5.1 [26] Let $f(x)$ be a linearized polynomial with $\operatorname{deg}_{q}(f) \leq n-1$. Then $\mathcal{C}_{f}$ is an $\mathbb{F}_{q}$-linear MRD-code with parameters $(n, n, q ; n-1)$ if and only if $U_{f}$ is a maximum scattered $\mathbb{F}_{q}$-subspace of $\mathbb{F}_{q^{n}} \times \mathbb{F}_{q^{n}}$, i.e., $f$ is a scattered q-polynomial.

Moreover, in [9], the authors prove that $\mathcal{C}_{f}$ is $\mathbb{F}_{q^{n}}$-linear on the left, i.e. $L\left(\mathcal{C}_{f}\right) \simeq \mathbb{F}_{q^{n}}$, and any MRD-code with parameters $(n, n, q ; n-1)$ with left idealizer isomorphic to $\mathbb{F}_{q^{n}}$ is equivalent to $\mathcal{C}_{f}$, for some scattered $q$-polynomial $f(x)$, in [9, Proposition 6.1]. Finally, we recall the following result concerning the equivalence.

Theorem 5.2 [26] If $\mathcal{C}_{f}$ and $\mathcal{C}_{g}$ are two $M R D$-codes arising from maximum scattered subspaces $U_{f}$ and $U_{g}$ of $\mathbb{F}_{q^{n}} \times \mathbb{F}_{q^{n}}$, then $\mathcal{C}_{f}$ and $\mathcal{C}_{g}$ are equivalent if and only if $U_{f}$ and $U_{g}$ are $\Gamma \mathrm{L}\left(2, q^{n}\right)$-equivalent.

Therefore, if $\mathcal{C}_{f}$ and $\mathcal{C}_{g}$ are equivalent, one gets that the associated linear sets $L_{f}$ and $L_{g}$ are PГL $\left(2, q^{n}\right)$-equivalent. The converse statement does not hold in general, see [25, Section 4.1]. By the results in Sect. 3, we have that

$-U_{\psi_{n}^{(k)}}$ and $U_{s}^{1, n}$,

$-U_{\psi_{8}^{(k)}}$ and $U_{s, \delta}^{2, n}$,

- $U_{\psi}^{(k)}$ and $U_{s, \delta}^{3,8}$

give rise to pairwise inequivalent MRD-codes for any compatible $k$. Then, to conclude the equivalence issue, we show the following

Proposition 5.3 Let $t \geq 3$ and assume that $\psi_{n}^{(k)}(x)$ is scattered, $1 \leq k<n$. Then the $\mathbb{F}_{q}$-subspaces $U_{\psi_{n}^{(k)}}$ and $U_{s, \delta}^{2, n}$ are not equivalent under the action of $\Gamma \mathrm{L}\left(2, q^{n}\right)$.

Proof Suppose that $U_{\psi_{n}^{(k)}}$ and $U_{s, \delta}^{2, n}$ are $\Gamma \mathrm{L}\left(2, q^{n}\right)$-equivalent. This is equivalent to suppose that $U_{\psi_{n}^{(k)}}$ and $U_{s, \delta}^{2, n}$ are GL $\left(2, q^{n}\right)$-equivalent. Furthermore, since, by Proposition 3.1, $U_{\psi_{n}^{(k)}}$ and $U_{s, \delta}^{2, n}$ are $\Gamma \mathrm{L}\left(2, q^{n}\right)$-equivalent if and only if $U_{\psi_{n}^{(n-k)}}$ and $U_{s, \delta}^{2, n}$ are $\Gamma \mathrm{L}\left(2, q^{n}\right)$-equivalent, we may suppose $k<t$. Then, let $\left(\begin{array}{ll}a & b \\ c & d\end{array}\right)$ be an invertible matrix in $\mathbb{F}_{q^{n}}^{2 \times 2}$ such that for any $x \in \mathbb{F}_{q^{n}}$ there exists $z \in \mathbb{F}_{q^{n}}$ such that

$$
\left(\begin{array}{ll}
a & b \\
c & d
\end{array}\right)\left(\begin{array}{c}
x \\
\psi_{n}^{(k)}(x)
\end{array}\right)=\left(\begin{array}{c}
z \\
\delta z^{q^{s}}+z^{q^{n-s}}
\end{array}\right) .
$$

In particular, one obtains that for any $x \in \mathbb{F}_{q^{t}}$

$$
c x+d x^{q^{k(t-1)}}=\delta\left(a x+b x^{q^{k(t-1)}}\right)^{q^{s}}+\left(a x+b x^{q^{k(t-1)}}\right)^{q^{n-s}} .
$$

That is, any $x \in \mathbb{F}_{q^{t}}$ is a root of the polynomial

$$
c x+d x^{q^{t-k}}-\delta a^{q^{s}} x^{q^{s}}-\delta b^{q^{s}} x^{q^{n-k+s}}-a^{q^{n-s}} x^{q^{n-s}}-b^{q^{n-s}} x^{q^{n-k-s}} .
$$


- Case 1: $s<t$. Then, the polynomial in (36) becomes

$$
c x+d x^{q^{t-k}}-\delta a^{q^{s}} x^{q^{s}}-\delta b^{q^{s}} x^{q^{e_{1}}}-a^{q^{n-s}} x^{q^{t-s}}-b^{q^{n-s}} x^{q^{e_{2}}},
$$

where $e_{1}$ and $e_{2}$ are the remainders of the divisions of $n-k+s$ and $n-k-s$ by $t$, respectively, and this polynomial is the null one.

Call $M_{1}, M_{2}, \ldots, M_{6}$ the monomials in (37).

- Case la. $s=k$. Since $k$ and $t$ are relatively prime, the integers $k, t-k$ and $e_{2}$ are distinct. Then, $M_{1}, M_{4}$ are of degree $q^{0}$;

$M_{2}, M_{5}$ are of degree $q^{t-k}$;

$M_{3}$ is of degree $q^{k}$;

$M_{6}$ is of degree $q^{e_{2}}$.

This implies $a=b=0$, a contradiction.

- Case 1b. $s=t-k$. Since $k$ and $t$ are relatively prime, the integers $k, e_{1}$ and $t-k$ are distinct. Then,

$M_{1}, M_{6}$ are of degree $q^{0}$;

$M_{2}, M_{3}$ are of degree $q^{t-k}$;

$M_{4}$ is of degree $q^{e_{1}}$;

$M_{5}$ is of degree $q^{e_{2}}$.

This implies $a=b=0$, a contradiction.

- Case 1c. $s \neq k$ and $k+s \neq t$. In this case, $M_{1}$ is the unique monomial of degree $q^{0}$, whence $c=0 ; M_{1}$ is the unique monomial of degree $q^{t-k}$, whence $d=0$, a contradiction.

- Case 2: $s>t$. Then, one may suppose that $s=t+r$ with $r<t$. Then, the polynomial in (36) becomes

$$
c x+d x^{q^{t-k}}-\delta a^{q^{s}} x^{q^{r}}-\delta b^{q^{s}} x^{q^{d_{1}}}-a^{q^{n-s}} x^{q^{t-r}}-b^{q^{n-s}} x^{q^{d_{2}}},
$$

where $d_{1}$ and $d_{2}$ are the remainders of the divisions of $n-k+r$ and $n-k-r$ by $t$, respectively, and this polynomial is the null one.

As before, call $N_{1}, N_{2}, \ldots, N_{6}$ the monomials in (38). Proceeding as in the previous case, a contradiction is obtained.

In view of Theorem 5.2, the following result summarizes Propositions 3.1, 3.3, 3.5, 3.6 and 5.3.

Theorem 5.4 Let $t \geq 3$ and $q$ odd if $t$ is even, or $q \equiv 1(\bmod 4)$ if $t$ is odd. Furthermore, let $1 \leq k<t$ be such that $\operatorname{gcd}(k, 2 t)=1$. Then, the code $\mathcal{C}_{\psi_{2 t}^{(k)}}(c f$. (35) (9)) is an $M R D$-code with parameters $(2 t, 2 t, q ; 2 t-1)$ not equivalent to any previously known MRD-code. The $\varphi(2 t) / 2^{1}$ codes obtained in this way are distinct up to equivalence.

Funding Open Access funding provided by Università degli Studi di Padova.

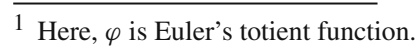


Open Access This article is licensed under a Creative Commons Attribution 4.0 International License, which permits use, sharing, adaptation, distribution and reproduction in any medium or format, as long as you give appropriate credit to the original author(s) and the source, provide a link to the Creative Commons licence, and indicate if changes were made. The images or other third party material in this article are included in the article's Creative Commons licence, unless indicated otherwise in a credit line to the material. If material is not included in the article's Creative Commons licence and your intended use is not permitted by statutory regulation or exceeds the permitted use, you will need to obtain permission directly from the copyright holder. To view a copy of this licence, visit http://creativecommons.org/licenses/by/4.0/.

\section{References}

1. Bartoli, D., Montanucci, M.: Towards the full classification of exceptional scattered polynomials. J. Combin. Theory Ser. A (accepted). arXiv:1905.11390

2. Bartoli, D., Zhou, Y.: Exceptional scattered polynomials. J. Algebra 509, 507-534 (2018)

3. Bartoli, D., Giulietti, M., Marino, G., Polverino, O.: Maximum scattered linear sets and complete caps in Galois spaces. Combinatorica 38, 255-278 (2018)

4. Bartoli, D., Zanella, C., Zullo, F.: A new family of maximum scattered linear sets in PG(1, $\left.q^{6}\right)$. ARS Math. Contemp. 19, 125-145 (2020)

5. Blokhuis, A., Lavrauw, M.: Scattered spaces with respect to a spread in PG(n, q). Geom. Dedicata 81, 231-243 (2000)

6. Csajbók, B.: Scalar $q$-subresultants and Dickson matrices. J. Algebra 547, 116-128 (2020)

7. Csajbók, B., Marino, G., Polverino, O.: Classes and equivalence of linear sets in PG(1, q $\left.{ }^{\mathrm{n}}\right)$. J. Combin. Theory Ser. A 157, 402-426 (2018)

8. Csajbók, B., Marino, G., Polverino, O.: A Carlitz type result for linearized polynomials. Ars Math. Contemp. 16(2), 585-608 (2019)

9. Csajbók, B., Marino, G., Polverino, O., Zanella, C.: A new family of MRD-codes. Linear Algebra Appl. 548, 203-220 (2018)

10. Csajbók, B., Marino, G., Zullo, F.: New maximum scattered linear sets of the projective line. Finite Fields Appl. 54, 133-150 (2018)

11. Csajbók, B., Zanella, C.: On the equivalence of linear sets. Des. Codes Cryptogr. 81, 269-281 (2016)

12. Csajbók, B., Zanella, C.: On scattered linear sets of pseudoregulus type in PG $\left(1, q^{t}\right)$. Finite Fields Appl. 41, 34-54 (2016)

13. Delsarte, P.: Bilinear forms over the finite field, with applications to codig theory. J. Combin. Theory Ser. A 25, 226-241 (1978)

14. Koetter, R., Kschischang, F.R.: Coding for errors and erasures in random network coding. In: Proceedings of 2007 IEEE International Symposium on Information Theory (ISIT 2007), Nice, France, 24-29 June 2007, pp. 791-795

15. Lavrauw, M., Polverino, O.: Finite semifields and Galois geometry. In: De Beule, J., Storme, L. (eds.) Current Research Topics in Galois Geometry. NOVA Academic Publishers, Hauppauge (2011)

16. Lavrauw, M., Zanella, C.: Segre embeddings and finite semifields. Finite Fields Appl. 25, 8-18 (2014)

17. Lidl, R., Niederreiter, H.: Finite Fields, volume 20 of Encyclopedia of Mathematics and Its Applications, 2nd edn. Cambridge University Press, Cambridge (1997)

18. Liebhold, D., Nebe, G.: Automorphism groups of Gabidulin-like codes. Arch. Math. 107(4), 355-366 (2016)

19. Lunardon, G., Polito, P., Polverino, O.: A geometric characterisation of linear $k$-blocking sets. J. Geom. 74, 120-122 (2002)

20. Lunardon, G., Polverino, O.: Blocking sets and derivable partial spreads. J. Algebraic Combin. 14, 49-56 (2001)

21. Lunardon, G., Polverino, O.: Translation ovoids of orthogonal polar spaces. Forum Math. 16, 663-669 (2004)

22. Lunardon, G., Trombetti, R., Zhou, Y.: On kernels and nuclei of rank metric codes. J. Algebraic Combin. 46, 313-340 (2017)

23. Lunardon, G., Trombetti, R., Zhou, Y.: Generalized twisted Gabidulin codes. J. Combin. Theory Ser. A 159, 79-106 (2018) 
24. Marino, G., Montanucci, M., Zullo, F.: MRD-codes arising from the trinomial $x^{q}+x^{q^{3}}+c x^{q^{5}} \in$

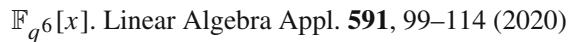

25. Polverino, O., Zullo, F.: Connections between scattered linear sets and MRD-codes. Bull. Inst. Combin. Appl. 89, 46-74 (2020)

26. Sheekey, J.: A new family of linear maximum rank distance codes. Adv. Math. Commun. 10, 475-488 (2016)

27. Silva, D., Kschischang, F.R., Koetter, R.: A rank-metric approach to error control in random network coding. IEEE Trans. Inform. Theory 54, 3951-3967 (2008)

28. Wu, B., Liu, Z.: Linearized polynomials over finite fields revisited. Finite Fields Appl. 22, 79-100 (2013)

29. Zanella, C.: A condition for scattered linearized polynomials involving Dickson matrices. J. Geom. 110, 50 (2019)

30. Zanella, C., Zullo, F.: Vertex properties of maximum scattered linear sets of PG(1, $\left.q^{n}\right)$. Discrete Math. 343, 111800 (2020)

Publisher's Note Springer Nature remains neutral with regard to jurisdictional claims in published maps and institutional affiliations. 